\title{
Application of BIM on Documenting Construction Defects
}

\author{
Ying-Mei Cheng
}

\begin{abstract}
This study aims to establish a system prototype using Autodesk Revit API to code the add-ins which display 3D elements with onsite quality defects. The result is a system that efficiently documents construction quality defects while improving communications regarding quality information.
\end{abstract}

Index Terms-Quality management, BIM, API, 3D.

\section{INTRODUCTION}

In recent years, the development of BIM is maturing due to joint efforts from the industry, government and academia in Taiwan, especially applications regarding benefit assessment, quantity calculations for concrete, rebar, and formwork, conflict management, and 4D scheduling. However, comparing to the above applications, the development of applications concerning other areas such as improvements in construction quality management seem inadequate. Quality management entails the establishment of policies and implementation of effective operation through a series of quality management system. The quality management framework for public works in Taiwan is shown in Fig. 1. Said framework is also called Three-level Quality Management System (TQMS). TQMS includes quality control, (QC, first level), quality assurance (QA, second level) and quality audit (third level). The contractor is in charge of quality control, project owners perform construction quality assurance and government authorities or Public Construction Commission (PCC) are responsible for quality audits. In addition, the public can serve the role of auditors and report construction defects through proper channels, which completes the framework and ensures project quality. This quality management system clearly defines the responsibilities of the owner and contractor. All participants of a project must adhere to the TQMS when carrying out operations related to public works unless specified otherwise. It is evident in this framework that the role of the first level, quality control, is the most fundamental and most eminent. Contractors may avoid wasting time and resources on redoing or re-inspecting the construction by properly manage the quality at this level. Lee et al. [1] stated that the onsite QA/QC tasks include reviewing and analyzing specified quality standards, testing and inspection procedures, and quality control practices; develop and monitor the QA programs of the organizations involved in construction operations; perform field testing and inspection; file documents and records to document management systems

Manuscript received November 4, 2016; revised October 23, 2016. This work was supported in part by Ministry of Science and Technology, R.O.C. (MOST 105-2221-E-163-001).

Ying-Mei Cheng is with the Department of Civil Engineering and Hazard Mitigation Design, China University of Technology, Taipei, Taiwan, ROC (e-mail: yingmei.cheng@msa.hinet.net). to keep evidence. The complexity of the tasks that construction companies need to deal with on site to reach certain quality is evident. If effective tools and support are available on site, they will help improve efficiency. In summary, the purpose of this research is to focus on the first level, quality control by contractors, and try to highlight the documentation and analysis of defects by the contractors with the use of Building Information Modeling (BIM).

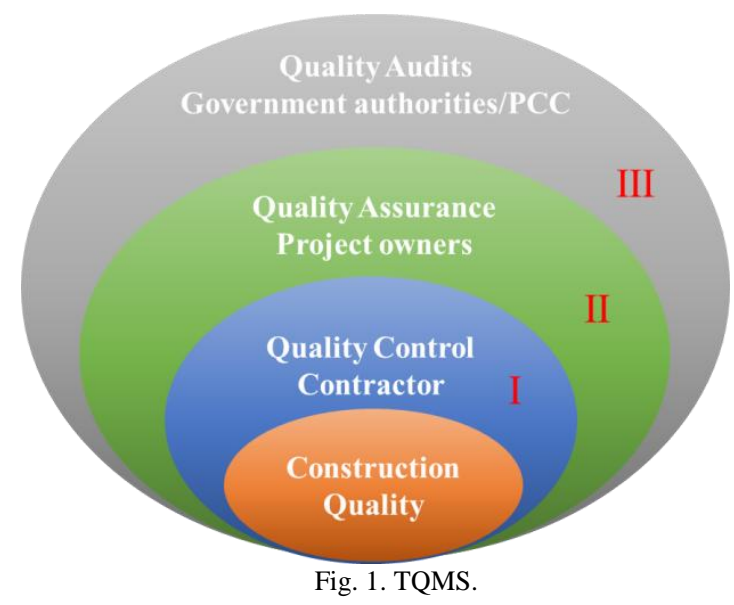

Most conventional construction companies document construction quality on site with a checklist. As the construction progresses, the number of checklist accumulates as well. As a result, the checklists are often poorly organized and managed, making them difficult to search through. The common structural defects identified during the third level, quality audit (PCC), as shown in Fig. 2, are crossed referenced with quality control from the first level. They also correspond to the elements in BIM. When the superintendent finishes construction inspection, the defects can be sent back to the database corresponding to BIM in a real-time fashion, which provides effective supports in the establishment of QC documents or review meetings. Therefore, the objective of this study is to use Autodesk Revit API to write the add-ins to present on site construction defects with $3 \mathrm{D}$ components. The research process is shown in Fig. 3. Once the research direction is determined and the literature review is completed, a new concept is proposed for the quality management operational system which combines the strengths of BIM and the traditional three-level quality management. Next, a BIM model for basic information will be established using Autodesk Revit for subsequent studies. In order to build the prototype of the information system, user needs must be defined first in order to design the user interface and process. The system prototype will then be constructed with Revit API and Microsoft Visual Studio C\#. 
01 main rebar/stimups binding is non-compliant 02 incorrect rebar size/ quantity/spacing/no detail drawing 03 inadequate lap length/ column rebar congestion due to splicing

04 the hook has inadequate angle or length of straight extension

05 not using spacer and cushion blocks, and the protective covers are non-compliant

06 not installing starter bars/not leaving adequate length/ spacing is too large

08 no strengthen rebar at openings or comer/ proportion is non-compliant

09 the beam-column connector anchor is not curved beyond the center of the beam

10 the anchorage of beam's rebar is not exceeding than 15 $\mathrm{cm}$

11 seriously corroded oil stains or concrete residue on the surface

12 the welding of steel cage is non-compliant 13 the coupler is poorly installed or highly rustic 14 other rebar defects
01 cold joints/ honeycombs/ pinholes/ bugholes/ blowholes 02 drying shrinkage

03 finished concrete surface are non-compliant(vertical/ horizontal variance)

04 debris are left on the concrete surface

05 expansion joint/ movement joint is inadequately placed, constructed, not installed

06 bursting during concrete pouring

07 high-flow concrete segregation and bleeding

08 sedimentation of aggregates in self-compacting concrete

09 other construction defects

01 overused/ does not meet the specifications

02 not organized/ not finished with concrete form oil applied with poor quality black oil

03 leakage of concrete grout

04 brace/horizontal or diagonal lacing defects

05 the formwork is tilted

06 opening/embedded conduits are not properly installed

07 not cleaned/ no access opening is installed

08 other formwork defects

Fig. 2. Quality defects

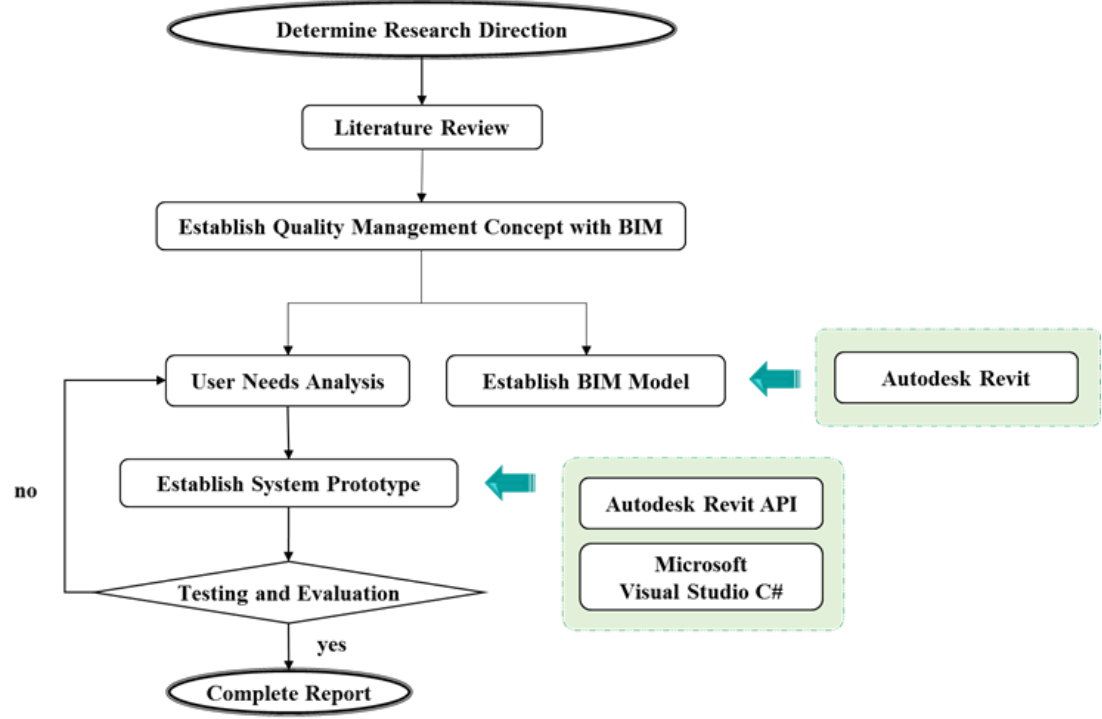

Fig. 3. Research process.

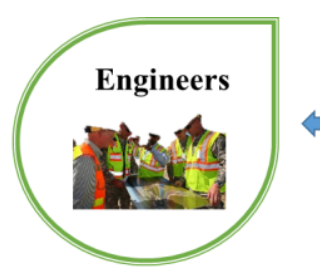

Functional Requirement

Quality defects Input

Quality defects Browse

Display $3 \mathrm{D}$ model with quality defects

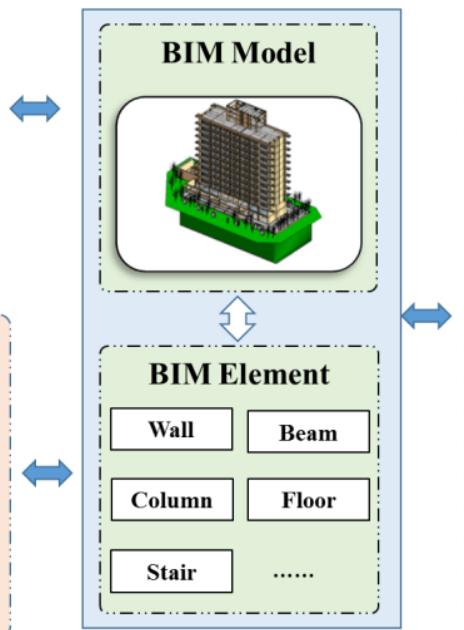

Fig. 4. User requirement. 


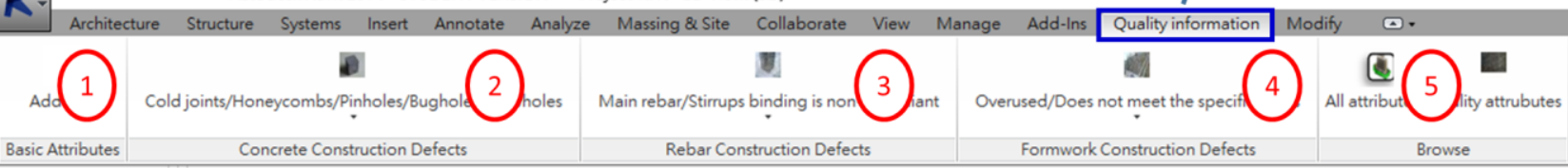

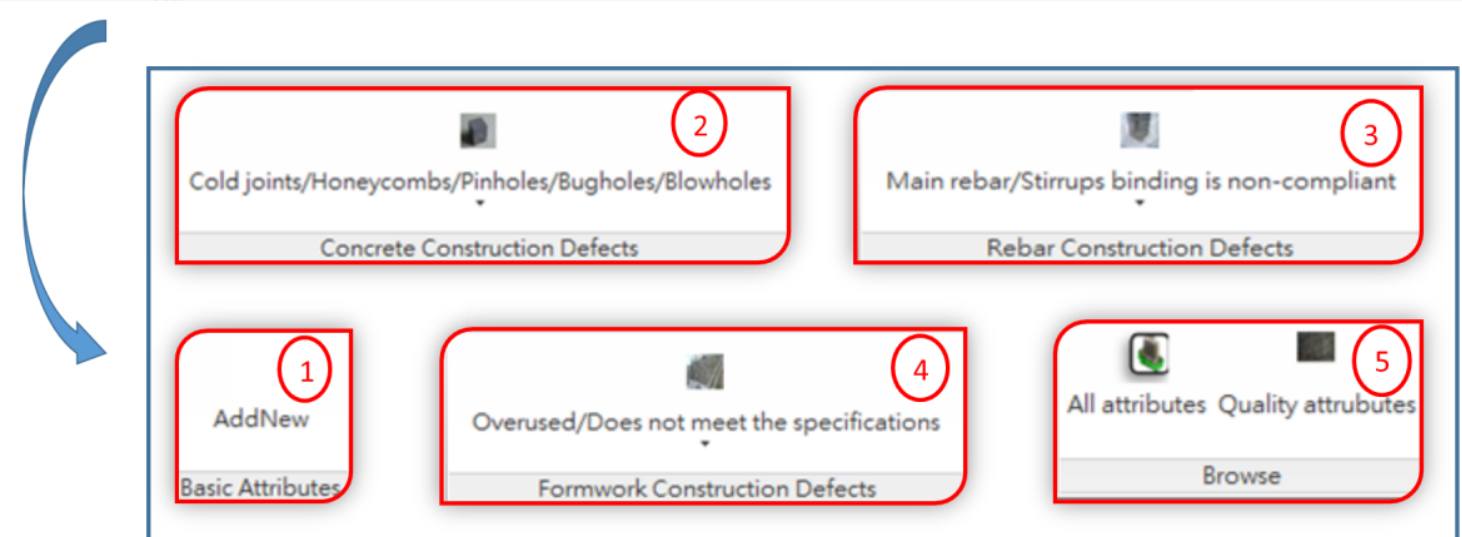

Fig. 5. System start-up screen.

Autodesk Revit 2014 - STUDENT VERSION - Project 1.Mt - 3D View. (3D)

$-0 \times$

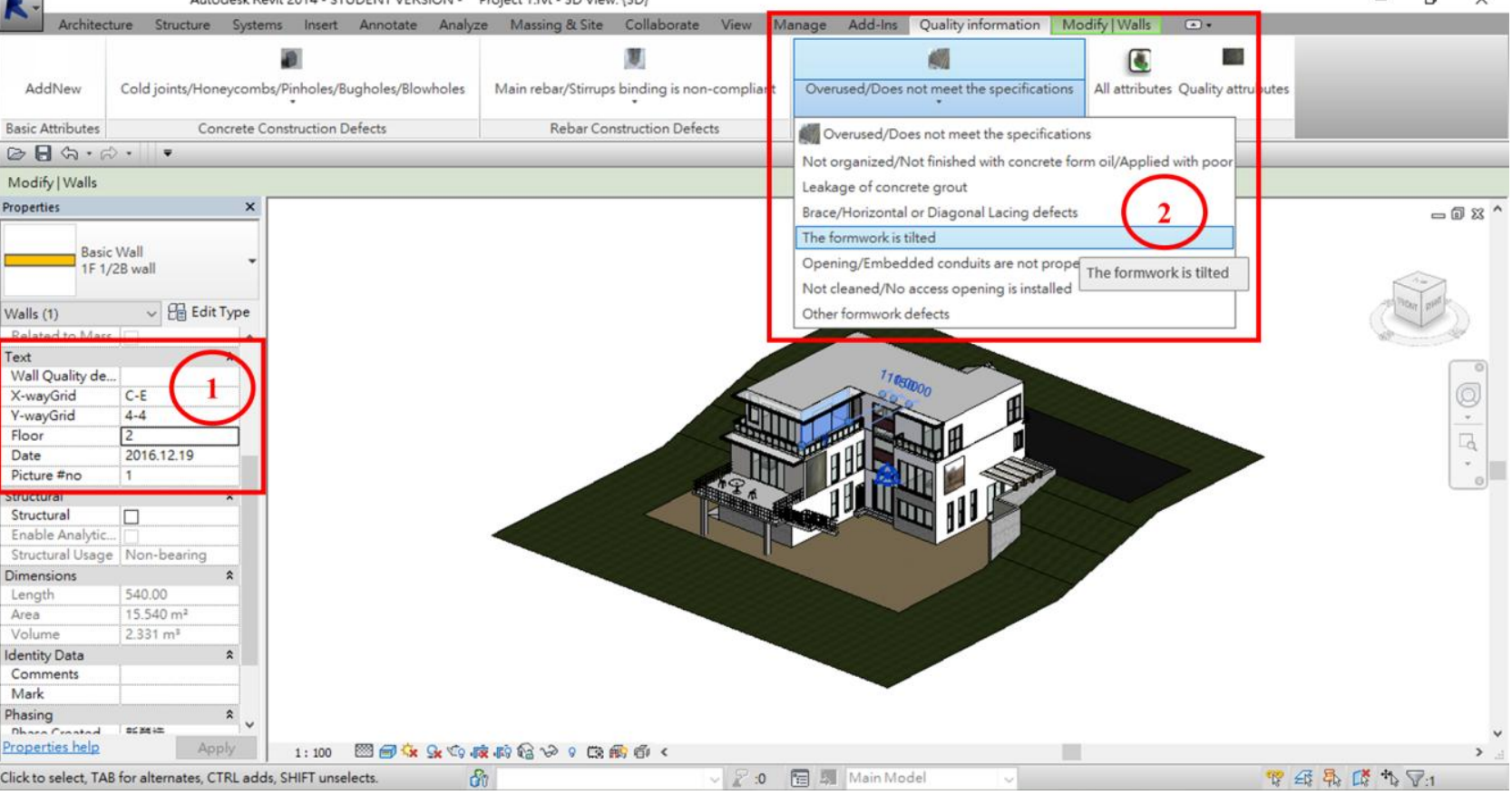

Fig. 7. Screening showing newly added quality defects.

\begin{tabular}{|l|l|}
\hline Properties & \\
\hline & \multicolumn{1}{|c|}{$\begin{array}{l}\text { Basic Wall } \\
\text { 1F } 1 / 2 \mathrm{~B} \text { wall }\end{array}$} \\
\hline & \\
\hline Walls (1) & \\
\hline Text \\
Wall Quality de... \\
\hline X-wayGrid \\
\hline Y-wayGrid \\
\hline Floor \\
\hline Date \\
\hline Picture \#no \\
\hline
\end{tabular}

Fig. 6. Basic attributes. 


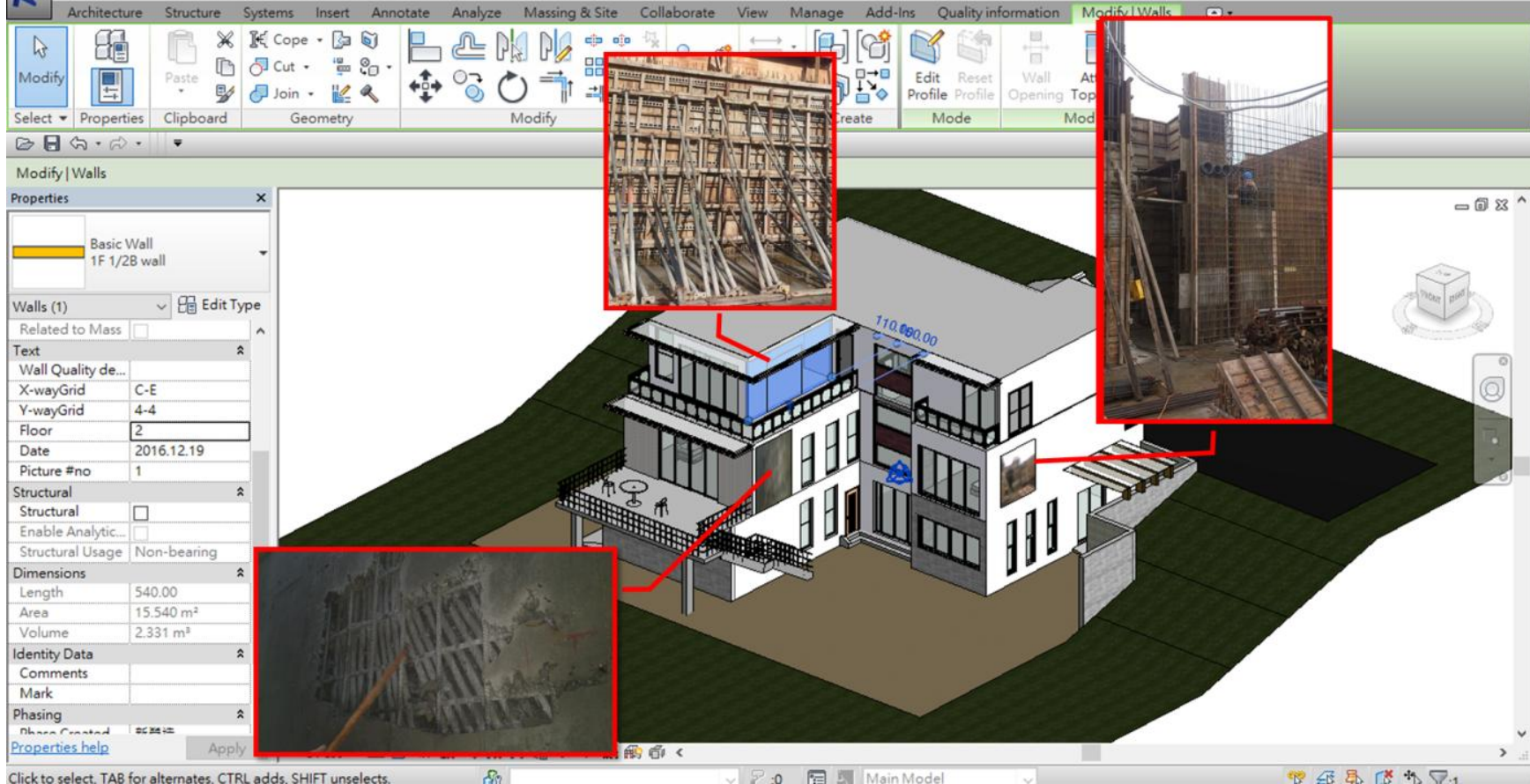

Fig. 8. Screen showing images of newly added quality defects.

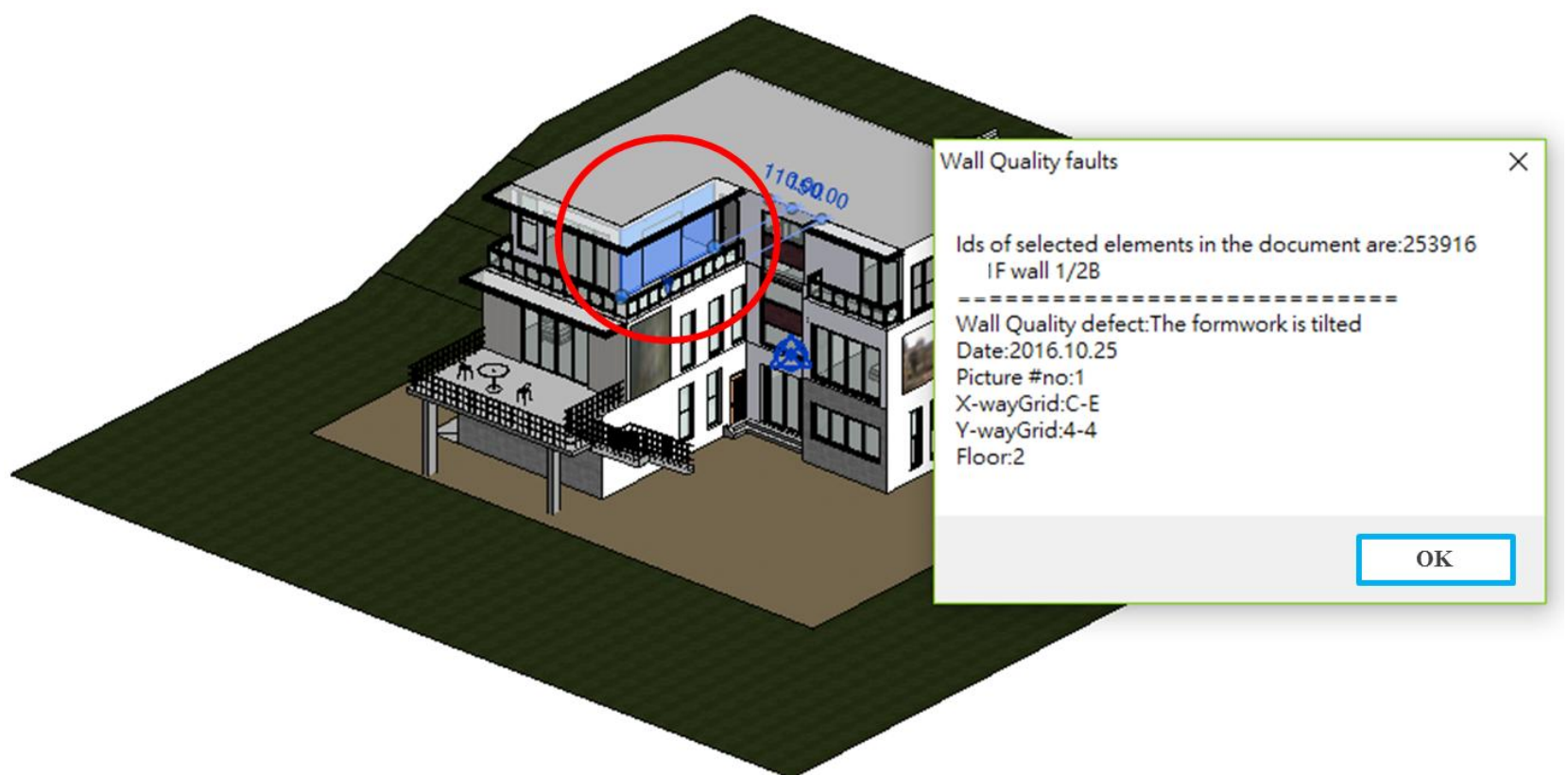

Fig. 9. Screen showing the search of quality information of element.

\section{LITERATURE REVIEW}

Love [2] mentioned that the benefits of implementing BIM include a reduction in construction costs, improved quality of design information, integration of project systems, data and teams, a reduced propensity for change orders, improved interoperability, and whole life-cycle asset management. Lee [1] also claimed that the successful application of the model-driven approach to QA/QC has great potential for communication, coordination, and collaboration in horizontal construction projects. Many studies discussed quantity evaluation, such as Chen [3], Fu [4], Guo [5], Cheng [6], and Monteiro [7]. However, literature concerning the use of BIM applications in the construction quality field is rather scarce.
Wang [8] developed an integrated system of BIM and Light Detection and Ranging (LiDAR) to come up with real-time onsite quality information collecting and processing for construction quality control. The authors discussed LiDAR-based real-time tracking system, BIM-based real-time checking system, quality control system, point cloud coordinate transformation system, and data processing system. The system prototype was developed for demonstrating the functions of flight path control and real-time construction quality deviation analysis. Kivimäki [9] proposed a new method where 3D design surfaces are produced and stored on a central collaboration cloud system. All measurement devices used on the site are integrated to the collaboration cloud via internet connection. All measurements made are 
immediately transferred over the internet to the cloud and automatically compared to designs to find height differences and color coded based on required tolerances. Chen [10] explored and discussed the advantages of 4D BIM for a quality application based on construction codes by constructing the model in a product, organization and process (POP) data definition structure. Wang [11] developed a systematic approach for BIM model fitness review through predefined standards.

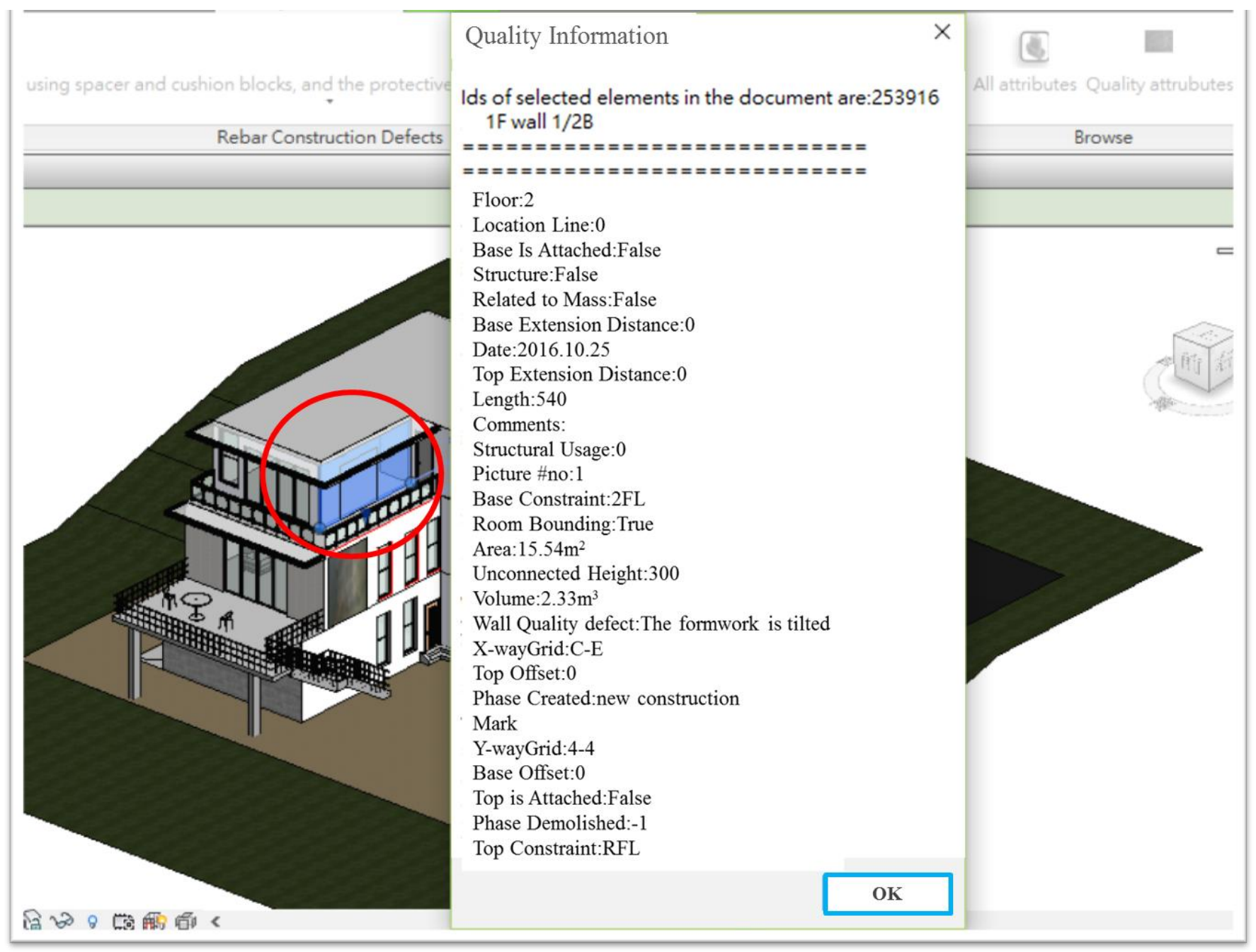

Fig. 10. Screen showing the search of all element information.

\section{RESEARCH METHODOLOGY}

This research adopts three-schema architecture of database development and uses Microsoft Visual Studio C\# to code the API of Autodesk Revit and build the quality system prototype. The three-schema architecture is an approach developed by the ANSI/X3/SPARC Standards Planning and Requirements Committee in 1978 to build the information system. It includes the external level, conceptual level and internal level. The goal of the three-level framework is to distinguish external functions and information from the format and operation of the information in the internal system. The internal information and external functions are independent from each other so that program developers can update and maintain the system without interfering with the user interface and the system framework. Details of each level are described below [12]:

\section{A. External Level}

This is the user analysis phase, which is to determine and define various user perspectives related to system development. The perspectives must fully convey users' needs toward the data and functions as a reference for subsequent data model development. The results are shown in Fig. 4.

\section{B. Conceptual Level}

At the conceptual level, user needs from the external level is integrated and categorized to establish a conceptual information model, which must include contents unrelated to the actual internal design, such as the format of how the information is documented as well as the attributes, interconnection, limitation, completeness, security and semantics of the information. The current stage of the study focuses on attaching quality parameters to the completed BIM elements. In order to accurately identify the parameters, they must be related to the element ID. Otherwise, the parameters are not interconnected for the time being. Table 1 shows the parameters added.

\section{Internal Level}

This level is directly related to the system developer, and the main task is to transform the conceptual information 
model into internal information model, including the information format, ranking approach, and index establishment. The developer also needs to choose appropriate software and hardware for system development. Microsoft Visual Studio C\# is used to write the API of Autodesk Revit during the study. This study will focus on walls to build the system prototype.

TABLE I: PARAMETER TYPE

\begin{tabular}{cc}
\hline \hline Parameter & Type \\
\hline Date & String \\
Floors & Integer \\
Grid-X & String \\
Grid-Y & String \\
Quality Faults & String \\
Picture \#no & Integer \\
\hline \hline
\end{tabular}

\section{SYSTEM PROTOTYPE}

Once Revit is open, the screen shows that quality information is added to the ribbon top. Click on it, and the screen shows Basic Attributes, Browse push button, and the split push button with Concrete construction defects, Rebar construction defects and Formwork construction defects, shown in Fig. 5. First-time user can start with the Add New push button to add basic attribute to the quality of an element and it will be added automatically in Properties Palette, as shown in Fig. 6.

The attributes include Date, Floor, Grid-wayX, Grid-wayY, Picture \#no and Quality Defect. These are the basic information for quality documentation. Once added, if quality defects occur on a particular wall during construction, users may use the system as they use Revit by adding basic quality information of the added elements in Properties Palette, and then use the split push button of Concrete construction defects, Rebar construction defects or Formwork construction defects based on actual conditions to correspond with the actual quality defects, as shown in Fig. 7. Once the defect is chosen, the system will highlight the interior and exterior of the wall in red and record the defect in the attribute of Quality Defect. Moreover, users may place the picture of defects on the element with Revit's Decal tool, and record the picture number in the Basic Attribute picture \#no column, as shown in Fig. 8.

When searching for quality defects of a certain element, click the particular element, followed by clicking the Quality Attribute push button under Browse, and the dialogue box will appear, as shown in Fig. 9.

To view all attribute information concerning a particular element, click the All Attribute push button under Browse, as shown in Fig. 10. The attribute information added is bound with the Revit database, and therefore, users can export the added quality information with the Revit database for analysis. Conclusion and Suggestion

This research uses BIM techniques to highlight its ability to document and analyze the defects for the contractor. A quality system prototype is established using Autodesk Revit API to code the add-in, which displays the 3D elements with onsite quality defects. At the current stage, this study focuses on the discussion of building the quality management system prototype for walls. Subsequent studies may consider other elements and further explore techniques related to automatic data export to allow users to further analyze the data or to produce printouts.

Basically, construction management includes quality, cost, schedule, safety and environmental management etc. Because that the BIM contain infinite possibilities, appropriately integrate it with construction management issues is the direction of future efforts.

\section{REFERENCES}

[1] N. Lee, T. Salama, and G. Wang, "Building information modeling for quality management in infrastructure construction projects," Computing in Civil and Building Engineering, pp. 65-72, 2014.

[2] P. E. D. Love, J. Matthews, I. Simpson, A. Hill, and O. A. Olatunji, “A benefits realization management building information modeling framework for asset owners," Automation in Construction, vol. 37, pp.1-10, 2014

[3] J. T. Chen, "Verification of quantity takeoff from BIM based models of reinforced concrete structure," M.S. thesis, Dept. Constr. Eng. Manage., Chung Hua University, Taiwan, R.O.C.2011.

[4] Y. M. Fu, "Application of BIM in quantity takeoff building materials works," M.S. thesis, Dept. Constr. Eng. Manage., Chung Hua University, Taiwan, R.O.C.2011.

[5] Y. F. Guo, "A study on feasibility of BIM-based costing in design stage," M.S. thesis, Dept. Constr. Eng. Manage., Chung Hua University, Taiwan, R.O.C. 2012.

[6] Y. M. Cheng, "Application of BIM on quantity estimate for reinforced concrete," in Proc. 2013 3rd International Conference on Civil Engineering, Architecture and Building Materials, May 25-26, Jinan, China.

[7] A. Monteiro and J. P. Martins, "A survey on modeling guidelines for quantity takeoff-oriented BIM-based design," Automation in Construction, vol. 35, pp. 238-253, 2013.

[8] J. Wang, W. Sun, W. Shou, X. Wang, C. Wu, H. Y. Chong, Y. Liu, and C. Sun, "Integrating BIM and LiDAR for real-time construction quality control," Journal of Intelligent and Robotic Systems, vol. 79, pp.417-432, August 2015.

[9] T. Kivimäki and R. Heikkilä, "Infra BIM based real-time quality control of infrastructure construction projects," International Association for Automation and Robotics in Construction (IAARC), 2014

[10] L. Chen and H. Luo, "A BIM-based construction quality management model and its applications," Automation in Construction, vol. 46, pp.64-73, 2014.

[11] J. Wang, X. Wang, W. Shou, J. Guo, and fL. Hou, "Development of BIM model fitness review system for modelling quality control," Computing in Civil and Building Engineering, pp. 577-584, 2014.

[12] G. W. Hansen and J. V. Hansen, Database Management and Design, Prentice Hall, 1992.

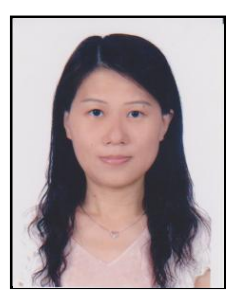

Ying-Mei Cheng was born in Taipei, Taiwan in 1970 She received the Ph.D. degree in construction management from the Department of Civil and Construction Engineering at National Taiwan University of Science and Technology in 2008.

From 1995 to 1996, she was an engineer of the BES Engineering Corporation. From 1996 to 1998, she was an engineer of Taiwan Housing \& Urban Development Bureau. Since 1999, she has been a faculty member of Civil Engineering and Hazard Mitigation Design, China University of Technology, Taipei, Taiwan, where she is currently an associate professor. Her research interests include (1) construction/ project management, (2) clustering theory (3) cost forecasting, and (4) BIM.

Dr. Cheng is a certified project manager (PMP) by PMI (Project Management Institute). She is a member of the PMI, and also is a member of the Chinese Society for Quality. 